\title{
IMAGING OF SNOW/ICE SUBSURFACE FEATURES FROM AIRBORNE SAR AT UHF, L AND X BAND. THE ONERA SAR CAMPAIGN IN SOUTH GREENLAND.
}

\author{
H.M-J. Cantalloube \\ ONERA, Université Paris Saclay F-91123 Palaiseau-France
}

\begin{abstract}
An acquisition campaign of the ONERA airborne synthetic aperture radar system RAMSES have been set up on the Greenland ice-shelf to assess the detectability of buried objects and subsurface feature through dry snow/ice cover. Three band were investigated UHF band, L band and X band, forecasting a better penetration at lower band, but an higher radar cross section for a given object/feature at higher band, thus a trade off for the optimal frequency band to use that was not clear prior to experiment.

Three sites were imaged, one in the accumulation zone North of the polar circle (East of Kangerlussuaq airport), one in the accumulation zone in a warmer southern area (East of Narsassuaq airport) and one in the intermediate zone between accumulation and ablation zones (plus a calibration zone and a front glacier close to Kangerlussuaq).

Processing of the data revealed that both UHF and X band are indeed providing complementing results (deeper bigger feature versus shallow smaller features), but intermediate L band combining both drawbacks of higher attenuation and lower features RCS did not seem to yield any further information.
\end{abstract}

Index Terms - Ground penetration radar, snow, ice, stereo radar imaging

\section{INTRODUCTION}

Satellite SAR imaging of icecaps and ice-shelves revealed an high radar return at $\mathrm{C}$ or $\mathrm{X}$ band, though the surface appears visually very smooth and homogeneous. SAR imaging of icy celestial bodies by space probes also revealed unexpectedly high surface reflection [1]. The supposed reason is that dry snow/ice is not very conductive, and large radio wave penetration allows buildup of radar cross section from several ice/snow layers.

This was corroborated by comparison between the ice shelf altitude derived from LIDAR measurements (such as ICEsat arctic terrain mapping) and the altitude derived from interferometric SAR (such as RadarSAT at C-band or TerraSAR at

\footnotetext{
Thanks to DGAC (French Air Directorate) for funding, to the Danish GEUS and Arctic Command for the valuable ground truth, target setup \& operational support. ICEsat DEM are provided by the Polar Geospatial Center under NSF OPP awards 1043681, 1559691 \& 1542736
}

$\mathrm{X}$-band) showing that the radar "surface" measured is some (tens of) meters below the optical surface [2].

The campaign presented here intends to use the higher spatial resolution and viewing angle flexibility of an airborne SAR sensor to investigate the though snow/ice imaging capability of synthetic aperture radar.

\section{EXPERIMENTAL SETUP}

The RAMSES airborne SAR system of ONERA was operated either at UHF+L bands in full polarisation mode with a range resolution of 63 and $100 \mathrm{~cm}$ resp, or at X-band alone in full polarisation with a range resolution of $20 \mathrm{~cm}$ ).

For calibration, a corner reflector was set close to the Kangerlussuaq airport (Fig. 1). Its position was measured with a few $\mathrm{cm}$ accuracy by differential GPS, allowing for fine estimation of the internal radar delays.

A similar corner reflector was buried under packed snow at one of the work area (area number 2, the "warm" accumulation zone). In this zone, a Luneburg lens with a partially emerging surface was set up (during the experiment, it ended up buried under $50 \mathrm{~cm}$ of fresh snow), as well as a few "artifacts" (hole dug and then filled back with packed snow and an ice lens made of a plastic sled filled with water buried at about $2 \mathrm{~m}$ depth).

\section{RESULTS FROM NADIR LOOKING (SOUNDING) SAR IMAGE MODE}

Though the antennae were pointed to the side $\left(45^{\circ}\right.$ down wing at UHF \& $\mathrm{L}$ band and 30 or $50^{\circ}$ at $\mathrm{X}$ band), significant side lobe (especially for $\mathrm{V}$ polarisation at $\mathrm{X}$ band) and the huge specular reflection at nadir allows nadir imaging (Fig. 2).

The sounding image at $\mathrm{X}$ band (with a depth resolution in vacuum of $20 \mathrm{~cm}$ ) shows a strong discontinuity at an apparent depth of about $2 \mathrm{~m}$. This is most probably the transition between the fresh snow fallen during the winter (acquisition is made early April) and the firn (percolated refrozen snow anterior to the previous summer). No further details appear at $\mathrm{X}$ band below this discontinuity. Note that the vertical scale inside the snow/ice cover should be downscaled by the refraction index, which is 1.25 to 1.35 in the upper layer and increases up to 1.76 for the solid ice at large depth. 


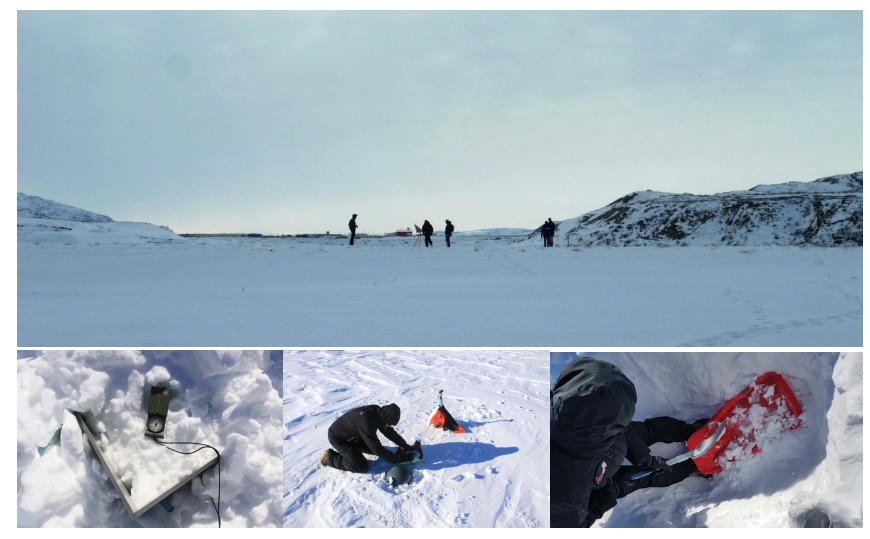

Fig. 1. Target setup: Calibrating corner reflector setup in Kangerlussuaq (top). Corner reflector during burial (bottom left), Luneburg sphere (bottom centre) and Ice lens during fabrication (bottom right)

Compared to $\mathrm{X}$ band, the $\mathrm{L}$ band (with a depth resolution in vacuum of $1 \mathrm{~m}$ ) do not show any more details (even the first discontinuity is almost beyond resolution). But UHF band (with a depth resolution in vacuum of $63 \mathrm{~cm}$ ) shows several discontinuities up to apparent depth of $38 \mathrm{~m}$ (the "index compensated" depth of $29 \mathrm{~m}$ for the lower discontinuity would indicate a 10 to 15 year old summer meltdown refrozen ice layer. The great meltdown of summer 2012 should be half way up).

Note on Fig. 2, that the sounding line at $\mathrm{X}$ band is offset by $200 \mathrm{~m}$ to the North, hence the surface profile do not match exactly that of the L and UHF bands (acquired simultaneously).

\section{RESULTS FROM SIDE LOOKING (USUAL) SAR IMAGE MODE}

Usual (side look focused) SAR images such as fig. 3 yield similar conclusions: on $\mathrm{X}$ band image, the edges of shallow crevices are visible, but deeper feature such as refrozen lakes are invisible. The ice cascade on the right edge (darker vertical zone) appears at $\mathrm{X}$ band, but the snow cover above ice is very shallow or even absent on this feature. The RCS of snow/ice cover at X band is indeed high: it ranges (for co-polar) approximatively from $-3 \mathrm{dBm} \mathrm{m}^{2} / \mathrm{m}^{2}$ to $+1 \mathrm{dBm^{2 }} / \mathrm{m}^{2}$ (for down wing angle from $25^{\circ}$ up to $65^{\circ}$ ) which is about $7 \mathrm{~dB}$ above that of a typical temperate forest.

At L band, the deeper crevices parallel to acquisition path becomes more visible and the buried frozen lakes appear blurred by the diffusion through the snow/ice upper layers.

Comparatively, the UHF image is considerably richer in deeper features: the tip of the air cavity in the crevices on the middle left of the image are buried under $40 \mathrm{~m}$ of snow/ice (compared with $2 \mathrm{~m}$ for the more recent one on the right) and the dense refrozen "floor" is around $65 \mathrm{~m}$ deep.

The UHF resolution, however, is less than a third of that of

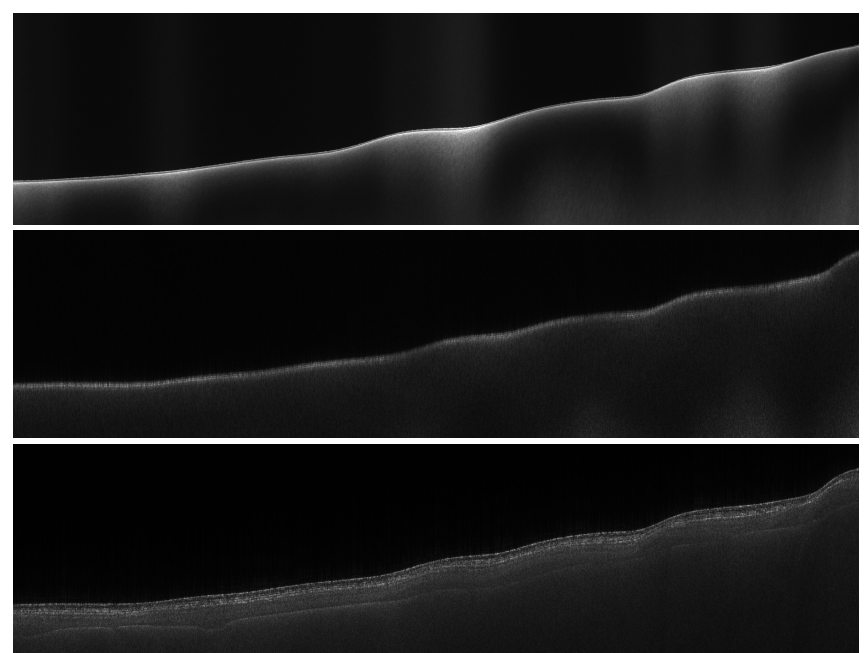

Fig. 2. Nadir (sounding) SAR mode images. X band (top), L band (middle), UHF band (bottom). Vertical span is $300 \mathrm{~m}$, length is $10 \mathrm{~km}$

the X-band, and RCS of small targets such as out setup is very low at this band.

On fig. 3 the white area at the top and bottom edges of $L$ and $\mathrm{X}$ band images corresponds to high noise to signal ratio where the aircraft roll motions shift the antenna pattern main lobe away from this edge of the swath. The hue vertical gradient on the UHF image is physical: the cross-polar to co-polar ratio increases with incident angle, which is expected from a "semi infinite random media" model ([3] for example).

The antenna orientation for the L-band was not precisely determined (due to its low interest), hence the antenna pattern compensation is poor for this image [This should be corrected for, in the final article].

\section{OBSERVATION OF BURIED TARGET}

Observation of the target buried on work area number 2 by GEUS yield unexpected results:

1. For the buried corner reflector, the measured RCS is from 25 to $35 \mathrm{~dB}$ below the one expected from an uncovered corner reflector of similar orientation at H-pol and more that $27 \mathrm{~dB}$ below at V-pol.

2. For the Luneburg lens, that was set up at the surface, but covered under $50 \mathrm{~cm}$ of fresh snow after the last radar acquisitions, there is a significant drop in RCS (32 to 38 $\mathrm{dB}$ ) between the first and the later acquisitions (when the lens was probably covered with snow). There is also, in the later images, a duplication of the echo in the range direction (fig. 4).

The reason is that a Luneburg Sphere is designed to act as a strong reflector when in a media of index close to 1 (as 

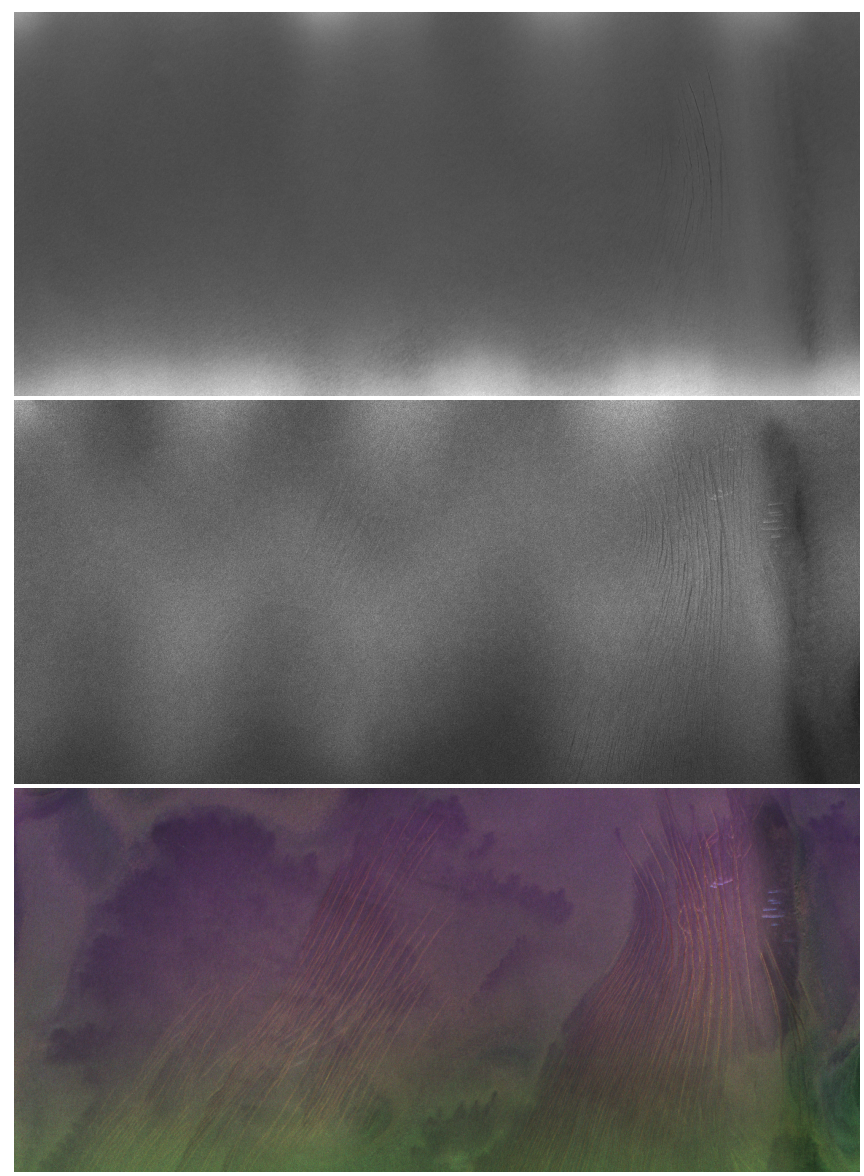

Fig. 3. Usual (side looking) SAR mode images. X band, Hpolarisation (top), L band H-polarisation (middle), UHF band $\mathrm{R} / \mathrm{G} / \mathrm{B}=\mathrm{Cross}-\mathrm{pol} / \mathrm{V}-\mathrm{pol} / \mathrm{H}-\mathrm{pol}$ (bottom).

air or vacuum), when buried in dense snow, the index of the surrounding media might be higher than the outer layer of the LSp (of index very close to 1 ). This has two consequences:

1. the snow/sphere interface behave as a big air bubble within the snow (hence a specular reflection at this surface).

2. the incoming waves are no more focused at the opposite metallized surface (the SPh becomes sort of farsighted) thus attenuating strongly the RCS of the target echo (which is localized somewhere beyond the bottom pole of the sphere opposite to the incoming radiation).

On fig. 4 the offset in position is due mis-compensation of stereo effect to calibrating target apparent depth: unlike the buried targets, the Luneburg sphere, intended to act as a reference point for ice drift, was emerging at the surface (at the beginning of the experiment).

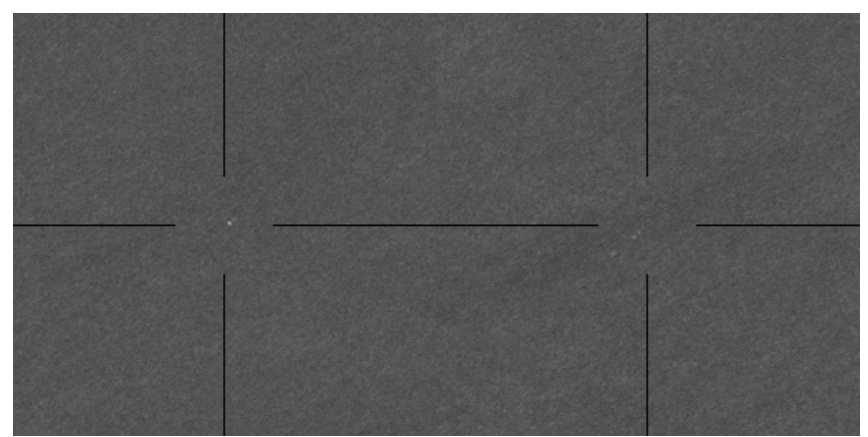

Fig. 4. Single acquisition images (6 looks $\times 4$ polar) of the Luneburg lens probably while not completely covered with snow (left) and later probably covered with about $50 \mathrm{~cm}$ fresh snow (right).

\section{MASSIVE MULTILOOKING FOR SHALLOW TARGET DETECTION}

Work area number 2 was imaged from 4 headings with 2 antenna pointing and 3 horizontal separations. Acquisitions from two headings were repeated with the aim of performing repeat-pass interferometry. However, the strong volume scattering made the repeated acquisition incoherent, with the inconvenient that interferometry (e.g. for ice drift or for target depth measure) was ruined, but the unexpected consequence is that the multilooking provides an higher equivalent number of looks (fig. 5-right) thus allowing a considerable smoothing of the clutter speckle without the resolution degradation of the conventional "spatial" speckle filters. This was favourable for detection of buried target with low RCS (consequence of the strong RCS damping described above).

Figure 5 shows on the left a detail of the native resolution $(20 \times 20 \mathrm{~cm})$ image. Detail area is $200 \times 265 \mathrm{~m}$ on the ground. The area is selected with shallow crevices for illustration purpose.

The image on the right of fig. 5 is the ENL for all acquisitions on work zone number 2 at a 100 times larger scale (full area is $20 \times 20 \mathrm{~km}$ ). The detail image is situated in the upper right of the white area.

Thermal \& system noise is about $20 \mathrm{~dB}$ below the clutter level for a SAR image computed in the middle of the main lobe in the antenna pattern, but when we compute 6 looks per channel and acquisition (a total amount of 432 looks for example on work zone number 2), the extreme looks angle correspond to image illumination of about $15 \%$ of main lobe level, and the Doppler ambiguities are some 7 times stronger. We thus mix images with marginal $(0.5 \%)$ noise + ambiguities together with images with about $50 \%$ noise + ambiguities. The combination law is derived from [4], with the extra twist that the clutter RCS is known (and not recursively estimated) and thus is directly injected into the algorithm.

Combination law is also slightly altered for compositing the 


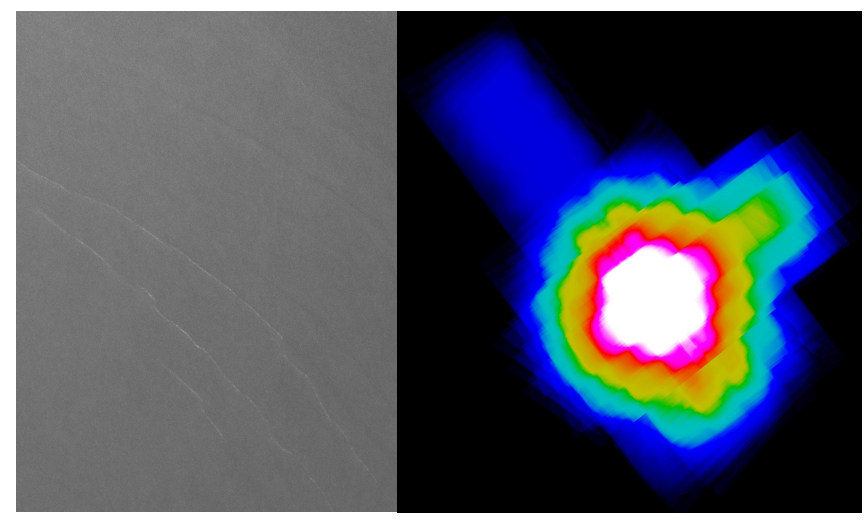

Fig. 5. Sample of composite image at $X$ band showing very low clutter speckle (left). Equivalent number of looks for the full work zone number 2 (right, false colour: yellow $=100$ looks, white $=$ above 200 looks).

reciprocal polar channel ( $\mathrm{Hv}$ and $\mathrm{Vh}$ ) because the Doppler (and left-right) ambiguities themselves reciprocal are coherent between the two images (unlike the thermal \& system noise). This explains also why the combination of 432 single look images ( 18 acquisitions $\times 6$ looks $\times 4$ polar channels) results in a maximum ENL slightly above 200 (fig. 5).

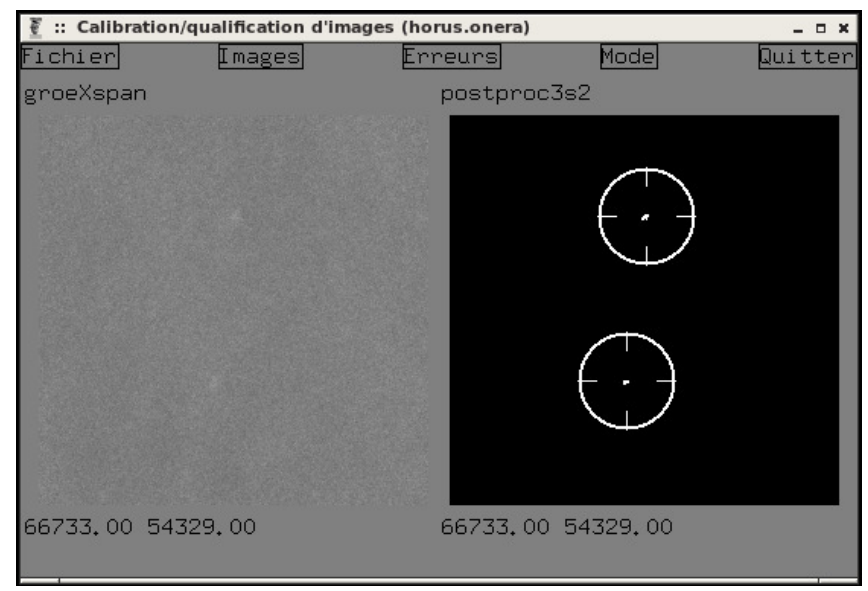

Fig. 6. Detection of the target setup ice lens (plastic sled filled with ice at the top of the window) and an empty hole packed with snow (bottom) on a composite image of all acquired $\mathrm{X}$ band images.

Combination of images from several headings (illumination directions) however, requires compensation of the stereo effect (consequence of the depth of the imaged feature). Because of refraction index of the snow/ice cover, the apparent stereo depth is different from the one deduced in the absence of refraction. Simplistic modelling by an homogeneous volume of constant index representative of the upper layers (1.25 to 1.35 ) showed that the transverse mis-positioning is below the $20 \mathrm{~cm}$ resolution cell, but the apparent/actual depth varies strongly with incident angle (from the refractive index in sounding mode, increasing with grazing incidence). Without knowledge of actual refractive index variation in the upper layers during acquisitions (there was a strong snow fall of an estimated $50 \mathrm{~cm}$ during the 5 days separating the North/South and the East/West acquisitions at X-band) the apparent depth used for image registration was adjusted using a calibrating known target (yielding an apparent depth of $3.4 \mathrm{~m}$ for the earlier acquisitions at $60^{\circ}$ incidence and $1.9 \mathrm{~m}$ for the later acquisitions at $40^{\circ}$ incidence).

\section{CONCLUSION}

Airborne SAR imaging at $\mathrm{X}$ and UHF band are a valuable tool for ice-shelf sub-surface observation: Remote observation of large features at UHF band to a depth of $50 \mathrm{~m}$ and of small objects at $\mathrm{X}$ band for depth of $3 \mathrm{~m}$ are possible. This requires, however, a large number of acquisitions and a significant computation load to yield results comparable to surface measurement with ground penetrating radars.

Due to the snow accumulation on the ice-shelf, this means that airborne SAR exploration has visibility horizon of roughly a couple of years for small features at X band and around 30 years for larger features at UHF bands. For example, the WW2 "lost squadron" present depth is estimated at around $100 \mathrm{~m}$ making it most probably beyond the range of this technique [5].

\section{REFERENCES}

[1] D. Campbell, J. Chandler, S. Ostro, G. Pettengill, and I. Shapiro, "Galilean satellites: 1976 radar results," Icarus, vol. 34, no. 2, pp. 254-267, 1978.

[2] J. Dall, "Insar elevation bias caused by penetration into uniform volumes," IEEE Transactions on Geoscience and Remote Sensing, vol. 45(7), pp. 2319-2324, November 2007.

[3] V.P. Tishkovets, P.V. Litvinov, and M.V. Lyubchenko, "Coherent opposition effects for semi-infinite discrete random medium in the double-scattering approximation," Journal of Quantitative Spectroscopy and Radiative Transfer, vol. 72, no. 6, pp. 803 - 811, 2002.

[4] H. Cantalloube and C. Nahum, "How to compute a multilook sar image?," in CEOS SAR Workshop, Toulouse, France 26-29 Oct 1999, ESA special publications, Ed., 2000, vol. 450, pp. 635-640.

[5] E. Krogager, "Results from the daloex 2015 campaign with f-sar in greenland," 2018, vol. 2018-June, pp. 608613. 